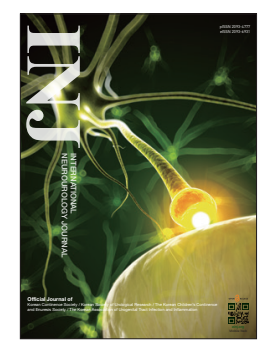

\title{
Applications of Basic Experimental and Clinical Research Using Biomarkers
}

\author{
Il-Gyu Ko (iD https://orcid.org/0000-0003-2696-6869 \\ Department of Physiology, College of Medicine, Kyung Hee University, Seoul, Korea \\ E-mail: rhdlfrb@naver.com
}

In the near future, the medical system is expected to undergo a paradigm shift due to advances in preventive medicine involving the early detection of diseases and treatment monitoring. In particular, the arrival of full-fledged personalized medicine for prediction and prevention is anticipated. The foundation of advances in personalized medicine lies in the rapid development of new techniques for detecting biomarkers.

A biomarker is an exogenous chemical, its metabolite, or the product of an interaction between a xenobiotic agent and a target molecule or cell that is measured within a compartment in an organism. The Biomarkers Definitions Working Group, composed of members from the U.S. Food and Drug Administration, National Institutes of Health, academia, and industry, has defined a biomarker as "a characteristic that is objectively measured and evaluated as an indicator of normal biological processes, pathogenic processes, or pharmacologic responses to a therapeutic intervention" [1]. Techniques related to nucleic acids and applied using DNA chips and microarrays have been used as the main analytical tools for biomarker screening. In medical research, the judicious use of biomarkers can improve the efficiency of drug development by identifying specific mechanisms that are relevant for selecting patients for treatment, dose optimization, and characterizing the effectiveness of compounds in clinical development. However, many studies of biomarkers have focused on a fractional approach to specific molecular indicators. Most such studies are at the level of searching for biomarkers, while very few studies have applied an integrated research approach to investigate clinical validity. This tendency is due to obstacles such as the high cost and difficulty of the required analyses, challenges in obtaining samples of specific diseases, and the problem of the clinical application of search parameters.

Recently, small-scale investigations into biomarkers have been actively conducted through a combination of clinical and basic research. Such studies have analyzed target biomarkers using tissues collected from patients and evaluated the efficacy of drugs in animal experiments using the proposed biomarkers. Miyake et al. [2] conducted a comprehensive gene expression analysis in human urothelial carcinoma of the bladder using a microarray and confirmed the presence of 2 types of collagen, COL4A1 and COL13A1. Based on this, an in vivo orthotopic experimental model of bladder tumors showed that intravesical treatment with siRNA targeting COL4A1 and COL13A1 inhibited the formation of the infiltrative pattern. In addition, similarly-designed studies have been performed of various urological diseases, such as chronic prostatitis/chronic pelvic pain syndrome [3], bladder outlet obstruction [4], overactive bladder [5], and prostatic hyperplasia [6].

Such studies involve an optimal combination of research methods, integrating clinical and basic research. Therefore, it is very important to conduct more research and to develop further our expertise regarding the analysis of biomarkers of urological diseases. These efforts may help us develop prevention strategies and personalized medicine for urological diseases.

- Conflict of Interest: No potential conflict of interest relevant to this article was reported. 


\section{REFERENCES}

1. Zhao X, Modur V, Carayannopoulos LN, Laterza OF. Biomarkers in pharmaceutical research. Clin Chem 2015;61:1343-53.

2. Miyake M, Hori S, Morizawa Y, Tatsumi Y, Toritsuka M, Ohnishi S, et al. Collagen type IV alpha 1 (COL4A1) and collagen type XIII alpha 1 (COL13A1) produced in cancer cells promote tumor budding at the invasion front in human urothelial carcinoma of the bladder. Oncotarget 2017;8:36099-114.

3. Roman K, Murphy SF, Done JD, McKenna KE, Schaeffer AJ, Thumbikat P. Role of PAR2 in the development of lower urinary tract dysfunction. J Urol 2016;196:588-98.
4. Lee T, Lim US, Kang DH, Jung HD, Kim H, Choi BH, et al. Nearnormalized gene expression profiles in bladder with detrusor overactivity in rats with bladder outlet obstruction after deobstruction. Int Neurourol J 2017;21:247-58.

5. Acevedo-Alvarez M, Yeh J, Alvarez-Lugo L, Lu M, Sukumar N, Hill WG, et al. Mouse urothelial genes associated with voiding behavior changes after ovariectomy and bladder lipopolysaccharide exposure. Neurourol Urodyn 2018;37:2398-405.

6. Ohya S, Niwa S, Kojima Y, Sasaki S, Sakuragi M, Kohri K, et al. Intermediate-conductance $\mathrm{Ca}^{2+}$-activated $\mathrm{K}+$ channel, $\mathrm{K}_{\mathrm{Ca}} 3.1$, as a novel therapeutic target for benign prostatic hyperplasia. J Pharmacol Exp Ther 2011;338:528-36. 